\title{
Light video game play is associated with enhanced visual processing of RSVP targets
}

\author{
Christina J. Howard, Robert Wilding and Duncan Guest \\ Nottingham Trent University \\ Christina.Howard@ntu.ac.uk \\ Room 4003, Chaucer Building \\ Nottingham Trent University \\ Burton Street \\ Nottingham \\ NG1 4BU \\ United Kingdom \\ Tel: +44 (0)115848 5556
}


Statement of ethics: The experiments reported here were conducted with the approval of the ethics committee of the School of Social Sciences at Nottingham Trent University and in accordance with the World Medical Association Helsinki Declaration as revised in October 2008. 


\begin{abstract}
There is mixed evidence that video game players (VGPs) may demonstrate better performance in perceptual and attentional tasks than non-video game players (NVGPs). The rapid serial visual presentation (RSVP) task is one such case, where observers respond to two successive targets embedded within a stream of serially presented items. We tested light video game players (LVGPs) and NVGPs on this task. LVGPs were better at correct identification of second targets whether or not they were also attempting to respond to the first target. This performance benefit seen for LVGPs suggests enhanced visual processing for briefly presented stimuli even with only very moderate game play. Observers were less accurate at discriminating the orientation of a second target within the stream if it occurred shortly after presentation of the first target, that is to say, they were subject to the 'attentional blink' $(\mathrm{AB})$. We find no evidence for any reduction in AB in LVGPs compared to NVGPs.
\end{abstract}




\section{Introduction}

Video games have become increasingly present in society and as a result their effects on cognition have become a popular topic for research in visual cognition. Although some suggest negative consequences of video games such as health risks and obesity (Pasch, Bianchi-Berthouze, van Dijk, \& Nijholt, 2009), many have identified potential positive aspects and motives for engaging in video games. Crawford and Gosling (2009) and Westwood and Griffiths (2010) for instance, have suggested that engagement in video games may provide rewarding opportunity for social interactions, whilst Connolly, Boyle, MacArthur, Hainey and Boyle (2012) and Orvis, Horn and Belanich (2009) have recently identified that the use of video games can have multiple benefits, ranging from higher levels of motivation to improvements in metacognitive strategies used in training situations. However some such as Gentile, Swing, Lim, and Khoo (2012) suggest video games can be damaging to sustained attention and others report very weak or null relationships between gaming and cognitive abilities (Unsworth et al., 2015), there are several lines of evidence suggesting the contrary, that video games can improve attention abilities (Castel, Pratt \& Drummond, 2005; Dye \& Bavelier, 2010; Dye, Green, \& Bavelier, 2009a; Dye, Green, \& Bavelier, 2009b; Green \& Bavelier, 2006a; Green \& Bavelier, 2003; Maclin et al., 2011; Strobach, Frensch \& Strubert, 2012; Vallett, Lamb, \& Annetta, 2013). However, the literature is mixed in terms of conclusions about possible differences between VGPs and NVGPs on various tasks and the nature of the processes underlying these differences.

The purpose of this paper is to examine differences in game players and NVGPs in their performance in a rapid serial visual presentation (RSVP) task, in particular by measuring performance in responding to either one or both of two targets in the RSVP stream. We focused on the RSVP task in particular because of what performance in reporting briefly presented targets can tell us about the temporal dynamics of perceptual processing. We sampled those with very moderate video game use (light video game players, LVGPs) to examine associations with light game play and attentional mechanisms. This is of interest since it is a relatively understudied group and also because it provides a much more conservative test for the associations between gaming and attentional differences: if differences are apparent even for very moderate gamers, then this lends much more weight to arguments that there is such a relationship. Some studies measure the causality of any such differences directly by means of a training manipulation (see below). Here we measure the association (and thus not causation) between moderate gaming and attentional processes only.

Superior performance has been demonstrated for VGPs compared to NVGPs in a number of visual and attentional tasks including multiple object tracking, task switching, contrast sensitivity, spatial resolution and backward masking. For instance, some have demonstrated greater endogenous control of attention and accordingly less exogenous attention capture in VGPs (Cain et al., 2014; Chisholm \& Kingstone, 2012), faster reaction times across a range of tasks (Castel, Pratt \& Drummond, 2005; Dye, Green \& Bavelier, 2009a), reduced backwards masking (Li et al., 2010) and higher quality feature representations during memory and tracking tasks (Applebaum et al., 2013; Sungur \& Boduroglu, 2012). In several of these studies, a causal role for game playing has been suggested by the use of a training manipulation after which NVGPs appear to perform more like VGPs (Green \& Bavelier, 2007; Green \& Bavelier, 2006a; Green et al., 2012; Strobach et al., 2012; Oei \& Patterson, $2013 ; 2015)$. Often the evidence for improved abilities in VGPs is mixed even within a paradigm. Further, whether or not gaming experience plays a causal role in these differences has been questioned (e.g. Boot, Blakely \& Simons, 2011; Kristjánsson, 2013) since a range of other factors such as motivation may underlie both gaming habits and any superior performance on such tasks.

In terms of attentional processes, there is some evidence for improved spatial attention in VGPs, for example in enumeration and multiple object tracking (MOT) tasks (Boot, Kramer, Simons, Fabiani, \& Gratton, 2008; Dye \& Bavelier, 2010; Green \& Bavelier, 2006b). There is also evidence that gamers may be more effective at selecting stimuli on the basis of their features - gamers are able to perform visual search faster than non-gamers (Castel, Pratt, \& Drummond, 2005; Hubert-Wallander et al., 2011), perform better in the useful field of view (UFOV) task (Green \& Bavelier, 2003; Feng, Spence 
\& Pratt, 2007), show improved spatial resolution of attention (Green \& Bavelier, 2007) and greater distractor filtering (Bavelier, Achtman, Mani \& Föcker, 2012) than non-gamers.

One paradigm that requires a high degree of attentional control is the RSVP task. The RSVP paradigm was first implemented by Raymond, Shapiro, and Arnell (1992). This method presents streams of letters or digits in quick succession, with each stream containing a first target (T1) and a second target (T2) requiring responses. In a single task condition, observers need only respond to T2, and in the dual-task condition, they must respond to both targets. The time between T1 and T2 is varied such that $\mathrm{T} 2$ can occur at different sequential positions (lags) after T1 within the stream. Raymond et al. (1992) were among the first to identify that with the RSVP, observers are often unable to detect T2 if it is presented between $180-450 \mathrm{~ms}$ after T1, a phenomenon they termed the 'attentional blink' (AB). The $A B$ is not simply a difficulty in responding accurately to $T 2$, but rather is the performance decrement going from single task trials (where no response to $\mathrm{T} 1$ is required) to dual task trials (where responses are made to both $\mathrm{T} 1$ and $\mathrm{T} 2$ ). The $\mathrm{AB}$ is a robust phenomenon and has been replicated many times (e.g. Arnell \& Jolicoeur, 1999; Chun \& Potter, 1995, 2001; Duncan, Martens, \& Ward, 1997; Dux \& Marois, 2010; Nieuwenstein, Potter, \& Theeuwes, 2009). The RSVP paradigm is useful for examining possible differences between VGPs and NVGPs since it provides sensitive measures of a range of processes including the rapid processing of visual information required for briefly presented targets.

Measures of performance for responding to targets in the RSVP stream (such as percent correct) measure the ability to perceive briefly presented stimuli. There is some evidence suggesting that VGPs do employ faster processing of sensory stimuli and potentially greater levels of temporal resolution in their perception of brief events. For example, Pohl et al. (2014) showed VGPs and NVGPs a number of masked semantic primes and measured the influence of these primes at various presentation durations. VGPs were more able to detect these masked primes than NVGPs, suggesting more rapid visual processing of these briefly presented mask stimuli. Further, they reported that VGPs experienced more priming than NVGPs for primes of short $(20 \mathrm{~ms})$ but not longer $(60 \mathrm{~ms})$ durations. This points towards faster perceptual processing of briefly presented stimuli in VGPS than NVGPS, since the primes had effects on VGPs' performance even when presented for a very brief period. These results are consistent with those of Li et al. (2010) who showed that action video gaming was associated with reduced backwards masking for target-mask asynchronies of around 50-150 ms. Applebaum et al. (2013) also argued that VGPs have greater sensitivity to visual information than NVGPs. They showed participants letter arrays for approximately $100 \mathrm{~ms}$ and then used a partial report method to query representations of the letter arrays after variable delays. They reported better performance for VGPs across the range of delays, interpreting this as superior sensitivity to the visual array in VGPs with no difference in the decay function in visual memory. Since the RSVP task involves accurately perceiving and responding to briefly presented stimuli, it therefore seems likely that LVGPs may outperform NVGPs in this task.

In terms of processes underlying the $\mathrm{AB}$ effect, the rate at which information is encoded into memory is thought to determine the severity of the blink. In this account, the AB occurs because of rapid forgetting of T2 (Chun \& Potter, 1995; Chun \& Potter, 2001; Giesbrecht, \& Di Lollo, 1998) since memory encoding resources are still taken up with the consolidation of $\mathrm{T} 1$ when $\mathrm{T} 2$ is presented. Another proposed mechanism that contributes to the $\mathrm{AB}$ is that of filtering out distractors in the RSVP stream. In an individual differences study examining why some people do not appear to experience the AB, Martens and Valchev (2009) showed that the presence of distractors within the stream harmed 'blinkers' much more than 'non-blinkers', suggesting that distractor filtering is linked to the emergence of the AB. Dux and Marois (2008) demonstrated that greater AB magnitudes were associated with greater priming from the distractor immediately preceding $\mathrm{T} 2$, suggesting that ineffective distractor filtering is a constituent of the $\mathrm{AB}$ phenomenon. Although it is possible to elicit the $\mathrm{AB}$ effect without a task switch between the responses required for T1 and T2 (Chun \& Potter, 2001; Jannati, Spalek \& Di Lollo, 2011; Kelly \& Dux, 2011), it is often used as part of the RSVP task and there is some evidence for reduced task switching costs in VGPs compared to NVGPs (Cain, Landau \& Shimamura, 2012; Colzato et al., 2010; Green et al.,2012). If VGPs do possess some task switching superiority, we might expect it to show up here as a reduced AB in LVGPs. 
To date, evidence on the relationship between gaming and RSVP tasks is mixed. Some studies report a reduction in the severity of the AB for VGPs compared to NVGPs. Green and Bavelier (2003) report a reduced attentional blink for VGPs and that action video game training also reduced the attentional blink in non-gamers. Dye and Bavelier (2010) showed reduced AB duration for children with gaming experience compared to other children. Oei and Patterson $(2013 ; 2015)$ showed that training in video gaming improved T2 detection during an RSVP task to an extent that depended on the characteristics of the game type used in the training. In a similar task, Mishra et al. (2011) asked observers to detect targets in one of three RSVP streams presented simultaneously at different locations in the visual field. VGPs outperformed NVGPs in terms of accuracy and reaction time although in this task targets were never presented in quick enough succession to induce an $\mathrm{AB}$. In contrast, Boot et al. (2008) found no difference between VGPs and NVGPs in the AB and did not find that training on action video games had any effect on the AB. Similarly to Boot et al., Cain et al. (2014) report no differences between VGPs and NVGPs in performance on the RSVP task. Murphy and Spencer (2009) present largely null results for a difference in AB between gaming groups including some evidence for slightly greater lag 1 sparing in VGPs compared to NVGPs, although this effect does not appear to be large enough to survive correction for multiple comparisons at different lags.

Given that light video game players are a relatively understudied group and that they provide a more conservative test for the presence of perceptual and attentional differences between game players and NVGPs, we sought to examine whether those with very moderate video game use (LVGPs) show improved visual processing for rapidly presented targets during an RSVP task and whether they show any differences in the attentional blink. We measured single and dual task performance separately at each of lags 1-8 in order to fully assess both the magnitude of the $A B$ and overall ability to respond to $\mathrm{T} 2$ under both task conditions. It is important to measure single and dual task performance separately for T2 since these represent two different tasks: accurate responding to T2 either with or without the additional demand of attending to and responding to T1.

\section{Method}

\section{Observers}

An opportunity sample of undergraduate students at the Nottingham Trent University volunteered to participate in the experiment. There were 43 observers in total with an age range of 19-44 (mean = 22.09) years, with the sample consisting of 14 males and 29 females. All observers reported having normal or corrected to normal vision.

\section{Apparatus and Stimuli}

The presentation of stimuli was adapted from that used in Raymond et al. (1992) which has been shown to be an effective measure of the AB on several other occasions (Dux \& Marois, 2010; Nieuwenstein et al., 2009). The first target (T1) was a white letter within the stream of otherwise black letters which observers attempted to identify. The second target, $\mathrm{T} 2$, was the letter ' $\mathrm{T}$ ' in one of four orientations, rotated by $0^{\circ}, 90^{\circ}, 180^{\circ}$ and $270^{\circ}$ and observers attempted to discriminate between these orientations. All stimuli were generated on a HP computer using Vision Egg software, and displayed on a 27 inch HP monitor, with a refresh rate of $100 \mathrm{~Hz}$. Observers viewed the monitor from a distance of $60 \mathrm{~cm}$, with letters being $1.05^{\circ}$ in height. All letters were presented in black on a grey background, with the exception of T1 which was a white letter.

\section{Procedure}

Each observer participated in two blocks of 96 trials and all observers performed a practice block of 10 trials until they were comfortable with the task before participation. Observers then participated in one block of single task trials and one block of dual task trials, the order of which was randomised across observers. On all trials, uppercase letters were presented in black on a grey background. Nontarget letters and $\mathrm{T} 1$ were drawn from a pool of all letters from the alphabet except for $\mathrm{T}$, and were 
presented in an RSVP at the same location in the centre of the screen, with the condition that no letter could appear twice in a single trial. Each letter was displayed for $20 \mathrm{~ms}$ separated by an $80 \mathrm{~ms}$ blank ISI, producing a presentation rate of $10 \mathrm{~Hz}$. The number of letters prior to T1 was randomised across all trials, ranging from 7-15 letters. T2 was presented at a lag of either 1, 2, 3, 4, 5, 6, 7, or 8 items after $\mathrm{T} 1$, such that a lag of 1 indicates no intervening items between $\mathrm{T} 1$ and $\mathrm{T} 2$. $\mathrm{T} 2$ was always a ' $\mathrm{T}$ ' with one of four orientations (rotated by either $0^{\circ}, 90^{\circ}, 180^{\circ}$ or $270^{\circ}$ ), with each orientation occurring with equal probability in any one of the 8 lags following T1. After T2, 8 non-target letters were presented (see Figure 1).

The dual task required observers to give unspeeded responses to two questions at the end of each stream of letters. They were first asked 'Which was the white letter?' and observers responded by pressing the appropriate letter on the keyboard. The second question was 'Which way up was the T?', to which observers responded to by pressing the arrow keys on the keyboard corresponding to the four orientations of the ' $\mathrm{T}$ '. Single task trials were identical to the tasks in the dual block, with the exception that there was no need to report the identity of the white letter (T1) and observers knew this from the start of the block.

After completing the RSVP task, observers were asked about their gaming habits over the last year and were classified as light video game players if they reported that they played action video games for one to five hours a week over this period. Observers were told that action video games were defined as games that include fast paced motion and looking out for multiple targets or threats, such as Halo, Call of Duty and Gears of War. Action video games were chosen since they have most often been associated with perceptual and attentional differences (e.g. Boot et al., 2008; Cain et al., 2014; Green \& Bavelier, 2003). No other types of gaming behaviour were recorded.

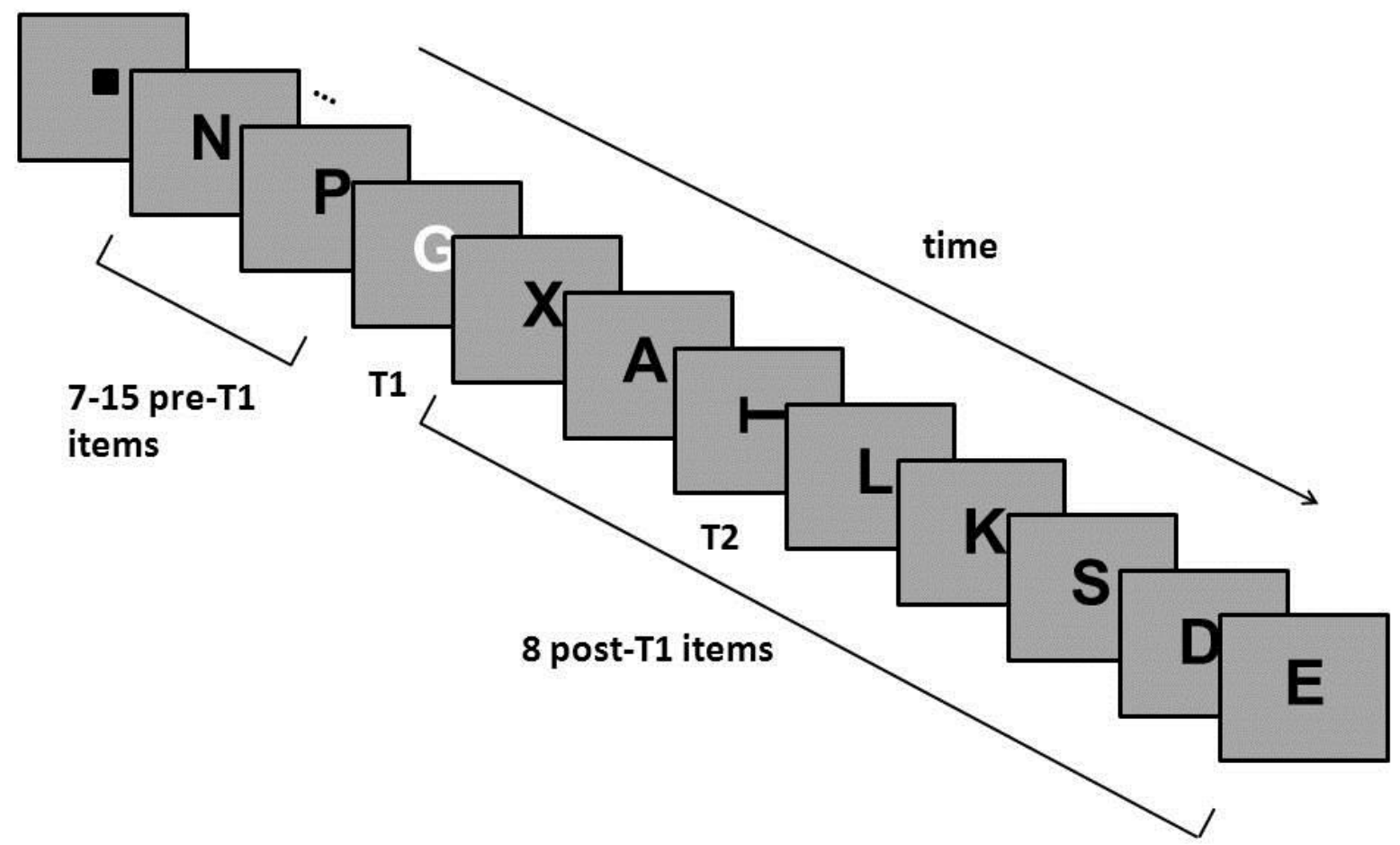

Figure 1. An illustration of the rapid serial visual presentation (RSVP) stimuli used in all trials. The first target in the stream (T1) was a white letter which observers were required to identify in dual task 
experiments only. The second target (T2) was always a ' $\mathrm{T}$ ' which was one of four randomised orientations $90^{\circ}, 180^{\circ}, 270^{\circ}$ and $360^{\circ}$ respectively. Each letter was presented for $20 \mathrm{~ms}$ with a blank $80 \mathrm{~ms}$ ISI between letter presentations. 


\section{Results}

Observers were classed as video game players (LVGPs) if they reported playing action video games for one to five hours a week over the past year. LVGPs consisted of 21 observers ( 8 female, 13 male) with an age range of 19-25 (mean $=21.23$ ) years. Non-video game players (NVGPs) consisted of 22 observers (21 female, 1 male) with an age range of 19-44 (mean $=22.95)$ years. Of those classed as LVGPs, twelve reported playing for one hour a week, three reported playing two hours a week, and six reported playing three hours a week. There was no relationship between number of hours of game play per week and single task performance $(\mathrm{r}(19)=0.27, \mathrm{p}=0.24)$ nor dual task performance $(\mathrm{r}(19)=$ $0.12, p=0.62)$. There was no relationship between number of hours of game play per week and mean difference between single and dual task performance $(r(19)=-0.02, p=0.93)$. There was still no relationship between these factors when just examining performance for lags $1-5(\mathrm{r}(19)=-0.02, \mathrm{p}=$ 0.92). Five women reported playing three hours a week in LVGP group and three reported playing for one hour a week. Of the LVGP men, one reported playing three hours a week, three reported playing two hours a week and nine reported playing for one hour a week. We were not able to examine performance in terms of potential gender differences due to the relatively low numbers of observers of each gender in each gaming classification.

To investigate between-groups differences, we calculated T2 performance on single and dual tasks as well as the single-to-dual performance differences at each lag and for the two groups separately, shown in Table 1 and Figure 2. LVGPs show generally better performance and slightly less effect of the dual task on performance compared to the single task baseline. Over the first three lag positions, NVGPs show a dip in performance of $11.50-23.01 \%$ whereas the LVGPs show a performance dip of $9.13-19.05 \%$.

Table 1. T2 discrimination performance in single and dual conditions for LVGPs and NVGPs.

\begin{tabular}{|c|c|c|c|c|c|c|c|c|c|}
\hline \multirow[t]{2}{*}{ Group } & \multirow[t]{2}{*}{ Task } & \multicolumn{7}{|c|}{ Lag } & \multirow[b]{2}{*}{8} \\
\hline & & 1 & 2 & 3 & 4 & 5 & 6 & 7 & \\
\hline \multirow{3}{*}{$N V G P$} & Single task & $92.06 \%$ & $92.06 \%$ & $92.06 \%$ & $92.82 \%$ & $92.46 \%$ & $94.05 \%$ & $92.46 \%$ & $91.67 \%$ \\
\hline & $\begin{array}{l}\text { Dual task } \\
\text { Performance }\end{array}$ & $80.56 \%$ & $69.05 \%$ & $74.60 \%$ & $86.90 \%$ & $88.10 \%$ & $91.67 \%$ & $91.67 \%$ & $96.83 \%$ \\
\hline & Difference & $11.50 \%$ & $23.01 \%$ & $17.46 \%$ & $5.92 \%$ & $4.36 \%$ & $2.38 \%$ & $0.79 \%$ & $-5.16 \%$ \\
\hline \multirow{3}{*}{$L V G P$} & Single task & $96.83 \%$ & $97.22 \%$ & $94.84 \%$ & $97.62 \%$ & $96.83 \%$ & $96.83 \%$ & $97.22 \%$ & $98.02 \%$ \\
\hline & $\begin{array}{c}\text { Dual task } \\
\text { Performance }\end{array}$ & $87.30 \%$ & $78.17 \%$ & $85.71 \%$ & $88.89 \%$ & $93.25 \%$ & $93.65 \%$ & $97.22 \%$ & $98.81 \%$ \\
\hline & Difference & $9.53 \%$ & $19.05 \%$ & $9.13 \%$ & $8.73 \%$ & $3.58 \%$ & $3.18 \%$ & $0.00 \%$ & $-0.79 \%$ \\
\hline
\end{tabular}

As shown in Figure 2, LVGPs generally performed better for T2 responses than NVGPs. Performance was worse overall for T2 discrimination under dual task than single task conditions, and this decrement was dependent on lag, with performance dipping with respect to single task conditions most between lags 1-3. 


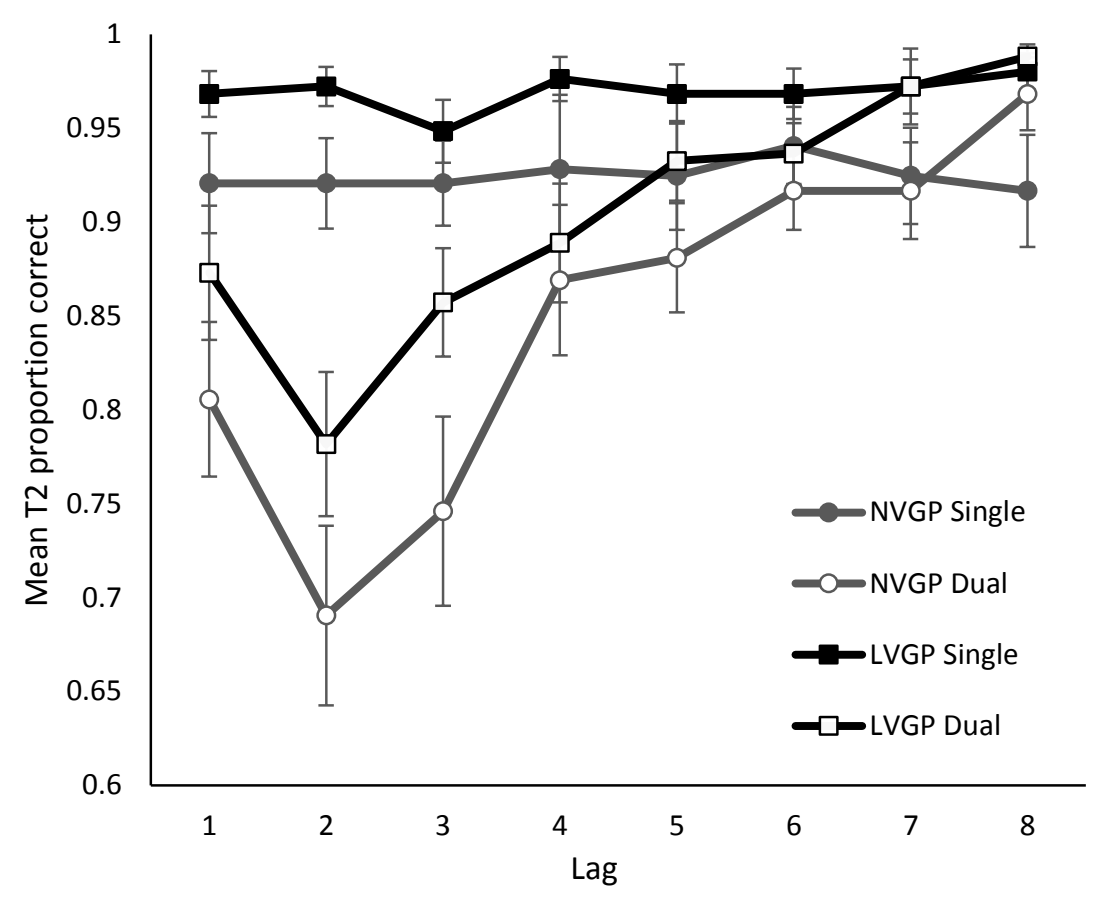

Figure 2. Performance of LVGPs and NVGPs in T2 discrimination in single and dual task conditions. Error bars indicate standard errors.

We explored T2 discrimination performance excluding dual task trials on which observers made incorrect T1 identifications in a 2 (Group: LVGP, NVGP) x 2 (Task: single, dual) x 8 (Lag: 1, 2, 3, 4, $5,6,7$, or 8) mixed measures ANOVA (as sphericity was violated Greenhouse-Geisser corrected effects are reported). This ANOVA identified a significant effect of task $(F(1,41)=8.586, p<0.01)$ with $\mathrm{T} 2$ detection in single task conditions being significantly better than detection in dual task conditions, thus confirming a detrimental effect of $\mathrm{T} 1$ processing on $\mathrm{T} 2$ processing. There was a significant effect of lag $(F(4.309,176.657)=19.221, p<0.001)$ indicating there were significant differences of performance between lags. In addition, there was a significant interaction between lag and task $(F(4.738,194.272)=20.211, p<0.01)$ with the effect of the dual task depending on lag, or in other words, the presence of an attentional blink. Note that because ANOVA looks for linear effects (e.g. a steady monotonic increase in dual task performance as lag increases) this is actually a very conservative test for the presence of a blink, since the characteristic dip shaped function produced by a blink will mask some of the effect of lag on dual task T2 performance. There was a significant between groups effect, with LVGPs performing better than NVGPs in general on T2 discrimination across task and lags $(F(1,41)=4.974, p=0.031)$.

Although T1 identification was only used to induce an attentional blink, we examined mean T1 performance for each group of observers. Mean T1 identification accuracy was $91.72 \%$ ( $\mathrm{SD}=7.10 \%$ ) for NVGPs and $93.25 \%$ ( $\mathrm{SD}=6.05 \%$ ) for LVGPs though these performance levels were not significantly different between groups $(\mathrm{t}(42)=0.646, \mathrm{p}=0.522)$. Looking at $\mathrm{T} 2$ discrimination performance overall (across single and dual task conditions), mean performance for NVGPs was $88.87 \%(\mathrm{SD}=8.25 \%)$ and for LVGPs was $93.65 \%(\mathrm{SD}=4.82 \%)$. Mean performance in $\mathrm{T} 2$ discrimination in single task trials was $92.71 \%(\mathrm{SD}=10.67 \%)$ for NVGPs and $96.92 \%$ for LVGPs $(\mathrm{SD}=4.55 \%)$. Under dual task conditions, $\mathrm{T} 2$ discrimination for NVGPs was $85.04 \%$ ( $\mathrm{SD}=12.38 \%$ ) and for LVGPs was $90.38 \%$ (SD $=8.80 \%)$.

There was no indication of a difference in $\mathrm{AB}$ between groups since there was no interaction between task and group $(F(1,41)=0.013, p=0.909)$ nor lag and group $(F(4.309,176.657)=0.826, p=0.518)$, neither was there any three way interaction between task, lag and group $(F(4.738,194.272)=1.338, p$ $=0.252$ ). Performing this ANOVA including all trials (including those with incorrect T1 responses) 
resulted in the same outcomes for all variables and interactions. However, ANOVA analyses are conservative estimates of $\mathrm{AB}$ effects since they only test for linear effects, where in fact the $\mathrm{AB}$ is dipper-shaped. To address this, we ran several post-hoc t-tests on T2 performance at different lags. Firstly, post hoc comparisons corrected for Type 1 errors (requiring $p<0.01$ ) were conducted to localise the $\mathrm{AB}$ effect in terms of lag. Paired t-tests were performed between single and dual task performance collapsed across groups and excluding trials with T1 reporting errors between lags 1-5 based on previous suggestions for the duration of the AB being up to 500ms (Dux \& Marois, 2010; Nieuwenstein et al., 2009). These revealed overall differences between single and dual task conditions on lag $1(t(42)=3.511, p=0.001), \operatorname{lag} 2(t(42)=6.204, p<0.001)$ and lag $3(t(42)=3.831, p<0.001)$, but not for lag $4(t(42)=1.983, p=0.054)$ or lag $5(t(42)=0.964, p=0.341)$. The duration of the overall $\mathrm{AB}$ here was therefore was a period of $100-300 \mathrm{~ms}$.

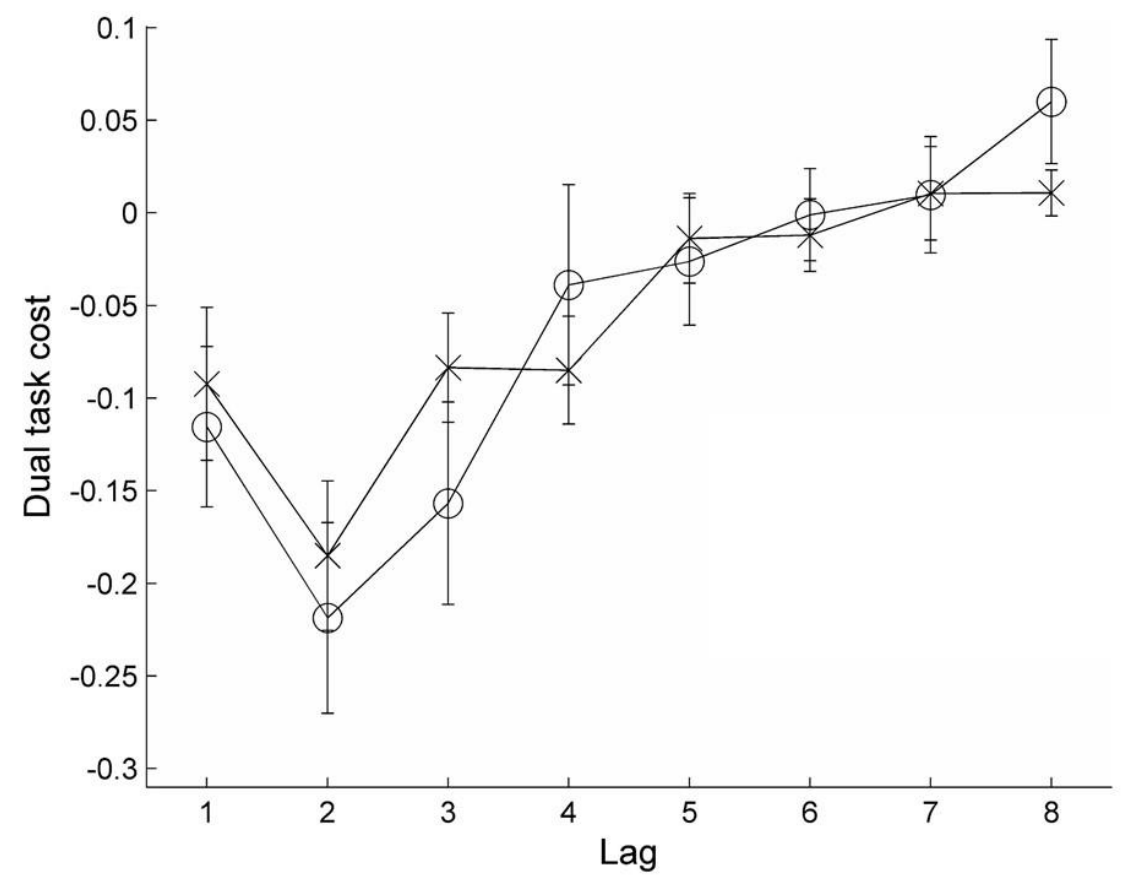

Figure 3: Attentional blink magnitude as measured by the difference between performance in single and dual task trials plotted separately for LVGPs (crosses) and NVGPs (circles). Only trials on which T1 was correctly reported are used here. Errors bars represent one standard error above and below the mean.

We performed five post-hoc comparisons between groups on the magnitude of differences between single and dual task performance (again excluding trials with incorrect responses to T1) as shown in Figure 3. For no individual lag between 1 and 5 did group affect the magnitude of differences between dual and single task performance (lag 1: $t(42)=0.385, p=0.702 ;$ lag $2: t(42)=0.511, p=0.612$; lag 3: $t(42)=1.163, p=0.251$; lag $4: t(42)=0.740, p=0.463$; lag 5: $t(42)=0.295, p=0.770)$. Therefore despite LVGPs showing numerically smaller differences between single and dual task performance at lags 1-3, these post-hoc tests confirmed the absence of any significant effect of gaming group on $A B$, even though LVGPs performed better on $\mathrm{T} 2$ discrimination overall.

We also assessed whether there were any differences in reaction times between groups. For T1 identification, NVGPs' mean RT was $1248 \mathrm{~ms}(\mathrm{SD}=170)$ and was $1271 \mathrm{~ms}(\mathrm{SD}=278)$ for LVGPs. These are fairly long reaction times but reasonable since observers had to choose and report using a standard keyboard which letter of the alphabet was presented as T1. There were no between groups differences in these RTs $(t(42)=0.321, p=0.750)$. Moving onto RTs for T2 discrimination under single task conditions, mean RT for NVGPs was $472 \mathrm{~ms}(\mathrm{SD}=116)$ and for LVGPs was $463 \mathrm{~ms}$ (SD $=169)$ and these were not significantly different $(t(42)=0.198, p=0.844)$. Under dual task 
conditions mean RT when making T2 reports for NVGPs was $358 \mathrm{~ms}(\mathrm{SD}=151)$ and for LVGPs was $455 \mathrm{~ms}(\mathrm{SD}=291)$ and these were not significantly different $(t(42)=1.40, p=0.170)$. Slightly counterintuitively, RTs were slightly shorter for dual task than single task conditions. This is not unexpected when we consider that during dual task trials, observers are first queried to report T1 identity and then afterwards are queried to report $\mathrm{T} 2$ orientation. Therefore observers are able to prepare their response to the $\mathrm{T} 2$ probe during the query and report of $\mathrm{T} 1$ that immediately precedes it.

In summary, LVGPs show better performance in the task of performing T2 reports when collapsed across single and dual task conditions. This is reflected in the higher proportion correct seen in Figure 2 for LVGPs than NVGPs. The presence of an attentional blink was evident in the detrimental impact of the dual task relative to the single task at shorter lags. However, we found no evidence for a difference in the magnitude of this blink for NVGPs and LVGPs. 


\section{Discussion}

The literature on differences in cognitive abilities between VGPs and NVGPs indicates various differences in spatial and temporal attention (e.g. Boot, Kramer, Simons, Fabiani, \& Gratton, 2008; Donohue, Woldorff \& Mitroff, 2010; Dye \& Bavelier, 2010; Green \& Bavelier, 2006b; Pohl et al., 2014). Whether or not they differ with respect to performance on the RSVP task and the attentional blink is less clear. Here, we show that even individuals with very moderate video game use (1-5 hours a week) exhibit better performance on the RSVP task in terms of overall ability to detect T2. This is striking since most of the literature arguing for improved attention abilities in VGPs has classed gamers as those with much greater levels of gaming behaviour than those we tested here. For example, Green and Bavelier (2003) classed individuals as VGPs if they had played for at least an hour a day for four or more days a week over the past six months. Boot et al. (2008) used a classification of playing for at least seven hours a week over the last two years, and Cain et al. (2014) used a definition of a minimum of five hours a week over the last six months. Therefore our finding of a difference in performance between light video game players and NVGPs provides a conservative test and evidence for the association between game play and elevated performance in visual cognition tasks.

Since LVGPs were more able to respond correctly to T2 across single and dual task conditions, they appear to benefit from more efficient perceptual processing of rapidly presented stimuli. The speed of visual information accrual is known to exhibit individual differences (Guest et al., 2015). VGPs show more semantic priming than NVGPs when semantic primes are very brief $(20 \mathrm{~ms})$ but not when they are presented for longer $(60 \mathrm{~ms}$ ) (Pohl et al., 2014), suggesting that more visual information is processed by VGPs during these very brief intervals. Another line of evidence to support this view comes from the useful field of view paradigm. Feng, Spence and Pratt (2007) show performance benefits for VGPs on this task, as do Dye and Bavelier (2010) for children and adults, although Boot et al. (2008) report a null result for group differences on this task. Whilst the UFOV is traditionally thought to measure sensitivity to stimuli across the spatial domain, the task typically uses very briefly presented stimuli (around $10-100 \mathrm{~ms}$ ) and a post-stimulus mask and hence this task may be measuring differences in the speed of perceptual processing. Similarly, Applebaum et al. (2013) briefly presented arrays of letter stimuli showing greater partial report accuracy for VGPs than NVGPs, which is consistent with faster processing of the stimuli. As mentioned above, a role for temporal aspects of attention is also broadly consistent with the findings from MOT (Boot et al., 2008; Dye \& Bavelier, 2010) where stimuli are defined by their spatio-temporal properties and therefore it is important not just to know where objects are, but where and when exactly.

Improved temporal resolution, or the ability to finely discriminate between successive events separated by brief intervals, is suggested by findings from Donohue, Woldorff and Mitroff (2010) who reported better performance for VGPs than NVGPs on temporal order and simultaneity judgements for cross modal visual-auditory stimuli. Donohue Woldorff and Mitroff (2010) show finer grained perception of timescales in temporal simultaneity and order judgements for VGPs, again potentially driven by faster perceptual processing speeds. Faster perceptual processing may allow for a more complete perceptual representation to have built up by the time memory consolidation processes have been freed up from $\mathrm{T} 1$. The higher the quality the representation of $\mathrm{T} 2$ when it enters the consolidation bottleneck, the more likely it will be correctly identified. Therefore faster perceptual processing speed will improve $\mathrm{T} 2$ report accuracy. Critically however, the quality of perceptual representations of the target cannot overcome the detrimental delay in consolidation caused by $\mathrm{T} 1$ processing, hence we would not expect perceptual processing speed to modulate the AB. That VGPs may process targets more effectively during an RSVP stream is supported by evidence from Mishra et al. (2011), who showed increased amplitudes of the P300 component in response to RSVP targets in VGPs than NVGPs. In the data presented here, no differences were observed between groups in T1 identification, probably because the T1 task was relatively easy - target 1 was a highly visible white letter in a black stream of letters and may have benefited from attention capture by luminance (e.g. Enns et al., 2001). In addition, letter identification is a highly practiced and familiar task and proceeds very rapidly ( $20 \mathrm{~ms}$; Adelman, 2011) which may have masked any facilitative effects of more efficient perceptual processing. 
It is also a possibility that LVGPs benefited from enhanced spatial attention here. Several sources of evidence point towards more effective spatial attention resources in VGPs than NVPs. For example, Green and Bavelier (2007) showed that VGPs were more able to tolerate smaller distances between targets and distractors in a crowding paradigm, suggesting enhanced spatial resolution in VGPs. Mishra et al. (2011) showed that when observers were asked to attend to one RSVP stream whilst ignoring two other streams presented at different spatial locations. Using steady-state visual evoked potentials, they found that VGPs showed enhanced suppression of distractor streams compared to NVGPs, which they accomplished by directing their spatial attention to the relevant stream. For other types of dynamic stimuli, the multiple object tracking task has been used several times to investigate differences in sustained spatial attention between VGPs and NVGPs. Boot et al. (2008) reported that VGPs could track three objects at a faster speed than NVGPs and Dye and Bavelier (2010) reported an ability to track more objects for both children and adults who self-reported as gamers. The same benefit appears to be present for the closely related task of judging the numerosity of objects as well as tracking (Green \& Bavelier, 2006b) although the findings in this respect are mixed since Boot et al. (2008) reported no significant effect of gaming on enumeration abilities. Improved spatial attention could have been used by LVGPs here to focus processing resources on centrally presented stimuli. Directed spatial attention is known to enhance stimulus detectability (Luck et al., 1994) and perceptual precision (Howard \& Holcombe, 2008) as well as the rate of visual processing (Carrasco \& McElree, 2001, Guest \& Lamberts, 2010, 2011). Therefore if gamers do possess an enhanced ability to direct spatial attention, we would expect target performance differences combined with no difference in blink magnitude as we report here.

We report no differences in reaction times between LVGPs and NVGPs which contrasts with the literature for VGPs which often shows RT advantages for gamers (e.g. Castel, Pratt \& Drummond, 2005; Dye, Green \& Bavelier, 2009a; Green \& Bavelier, 2003). This is not unexpected since our gaming group were light video gamers rather than those who engage in video gaming more regularly, however it does serve to highlight further the significant performance differences we report here even in the absence of any differences in speed of responding.

Our data show that LVGPs exhibited improved accuracy of responses to the orientation of T2 compared to NVGPs, consistent with faster perceptual processing and potentially enhanced spatially directed attention to stimuli. Perceptual processing of individual stimuli within the RSVP stream appears to be performed prior to processes giving rise to the AB. Several studies have shown that after perceptual processing of stimuli, a bottleneck of consolidation into working memory gives rise to the AB. Vogel, Luck \& Shapiro (1998) showed in an electrophysiological study that early visual responses to $\mathrm{T} 2$ were not modulated by the $\mathrm{AB}$, but that the later $\mathrm{P} 3$ component which is thought to reflect updating in working memory, was negatively affected by $\mathrm{T} 1$ reporting. It has also been shown that for 'blinked' word stimuli, semantic processing of T2 occurs despite the presence of an AB (Luck, Vogel \& Shapiro, 1996) which could only happen if early perceptual processes proceed unimpeded by T1 processing. Moreover, Arnell et al. (2006) reported that speeded perceptual identification responses to single stimuli predicted performance on $\mathrm{T} 1$ and $\mathrm{T} 2$ accuracy but not $\mathrm{AB}$ magnitude. This suggests that the bottleneck responsible for the $A B$ is not caused by a reduction in speed of perceptual processing. Since these studies show that the bottleneck seems to occur at a postperceptual stage, perceptual processes are candidate mechanisms for the improved $\mathrm{T} 2$ performance seen here in the absence of $\mathrm{AB}$ reduction. Temporary suppression models of the $\mathrm{AB}$ such as the 'boost and bounce' theory (c.f. Olivers and Meeter, 2008; Taatgen et al.,2009; Wyble, Bowman and Nieuwenstein, 2009) suggest that the $A B$ is caused by an enhancement of processing on detection of T1 which then causes compensatory inhibition of processing for subsequent items. The data reported here are consistent with this view, if LVGPs and NVGPs both experience the same facilitation and subsequent inhibitory mechanisms, but overlaid on a superior baseline level of processing for LVGPs compared to VGPs.

We identified an $\mathrm{AB}$ lasting in the period between lags 1-3, representing a time period of $100-300$ $\mathrm{ms}$, commensurate with prior findings that the $\mathrm{AB}$ has a duration of 180-500 ms (Dux \& Marois, 
2010; Martens \& Wyble, 2010; Nieuwenstein et al., 2009; Raymond et al., 1992). Although clear ABs were observed, like Boot et al. (2008) and Cain et al. (2014), we did not observe a difference in the size of the AB between gamers and non-gamers. Consistent with Green and Bavelier (2003) we show that LVGPs were better able to identify T2 than NVGPs, however, unlike them we find no evidence of a difference in $\mathrm{AB}$ between these groups. Of course a null result does not establish the lack of an effect and although non-significant, the differences between single and dual task performance were numerically smaller at lags 1-3 for LVGPs than for VGPs, Therefore it remains a possibility that we may have observed a difference in $\mathrm{AB}$ magnitude between our very moderate players and NVGPs perhaps with more statistical power. Alternatively it may be the case that only more extensive game play, or game play other than action video gaming is associated with significant changes in the magnitude of the AB. However it is noteworthy that we do not see a significant difference in blink magnitude despite clear differences in $\mathrm{T} 2$ detection between groups.

In the Green and Bavelier (2003) study, baseline T2 performance (identification in a single task situation where no T1 identification was required) differed between groups with VGPs being $7.7 \%$ more accurate. However, VGPs were near ceiling performance on this baseline single task $(95.6 \%)$ whereas NVGPs were not which may have led to the AB for NVGPs being more pronounced. Therefore, it was unclear whether the reduction in $\mathrm{AB}$ could have been at least in part due to ceiling effects observed for VGPs in T2 discrimination. Further, it is not clear whether the significant differences they report between groups at particular lags would survive corrections for multiple comparisons. Although we also had near ceiling performance for VGPs (96.92\%), critically, the same $\mathrm{AB}$ was observed as for NGVPs which would not be expected if this level of VGPs performance made it more difficult to produce an AB. Similarly, our results contrast with those of Dye and Bavelier (2010) who showed that attention returned faster to baseline levels for VGPs after the attentional blink for a group of children and young adults. However, this was less evident in their older age groups which may partially explain the different findings reported here. It is also not clear whether their measure of attentional return would yield the same results as a more traditional measure of the difference between single and dual task responses to T2. Furthermore, in the Dye and Bavelier (2010) study, a version of the RSVP task was adapted for children and there is a possibility that this adaptation made the task more sensitive to group differences. Items were a variety of coloured shapes and targets were isosceles triangles which could point in different directions. The colours for T1 and $\mathrm{T} 2$ items were kept constant for each observer e.g. red for T1 and blue for T2 and observers reported the direction in which they pointed. In theory this task can be performed simply by attempting to detect isosceles triangle shapes and then reporting the direction in which they point. There is a possibility that observers attempted to detect colour-shape conjunctions in the stream (e.g. a red isosceles triangle for T1) and that this two-feature colour-shape conjunction task somehow contributed to the difference seen between VGPs and NVGPs. In light of previous evidence that VGPs show enhanced visual search performance (Castel, Pratt, \& Drummond, 2005; HubertWallander et al., 2011) this seems a possibility.

As reviewed previously, there is evidence to suggest that the $\mathrm{AB}$ arises because of a bottleneck in consolidation of successive targets into memory (Arnell et al. 2010; Chun \& Potter, 2001; Colzato et al., 2007). We find no evidence for a reduction in the AB in LVGPs and therefore it is unlikely there were differences in these encoding processes between LVGPs and NVGPs here. Similarly, because distractor filtering has been implicated in explaining individual differences in the magnitude of the AB and we see no AB differences here, (Dux \& Marois, 2008; Martens \& Valchev,2009) it appears unlikely that LVGPs achieved their higher levels of performance for T2 on the basis of more effective distractor filtering. Lastly, the task used here involved a task switch since attentional control must be switched between reporting the identity of the white letter (T1,25AFC) and the orientation of the letter 'T' (T2, 4AFC). Since improved task switching in VGPs has previously been suggested (e.g. Cain, Landau \& Shimamura, 2012; Green et al., 2012; Strobach, Frensch \& Schubert, 2012) the fact that LVGPs did not show a reduced $\mathrm{AB}$ nor any apparent differences in the time course of the $\mathrm{AB}$ suggests no difference in task switching costs between the groups.

In summary, the improved T2 accuracy seen for LVGPs compared to NVGPs suggests that LVGPs may enjoy faster perceptual processing of stimuli. There is a possibility that they may be also able to 
direct spatial attention towards the RSVP stream more effectively, which in turn, may be associated with speeded perceptual processing. We categorised participants as LVGPs here if they reported playing action video games for one to five hours a week over the past year. If we had used a stricter criterion it is possible that any gaming effects would have shown up more clearly. However the fact that we do detect an overall performance difference here shows that our two groups of participants did differ in the abilities on the task, even in the absence of measured differences in AB.

Although we report a statistical association here between T2 reporting and very moderate gaming behaviour, we make no claim here about whether there is a causal role for video game playing on performance in attentional tasks as identified by some (e.g. Feng, Spence \& Pratt, 2007; Green \& Bavelier, 2006a; Green \& Bavelier, 2007; Green et al., 2012; Strobach, Frensch \& Schubert, 2012). Indeed it remains possible that the relationship identified here is either non-causal or is even causal in the opposite direction, for example if it is improved attention skills that increase the likelihood of enjoyment of video gaming and not the other way around. Other intervening variables and such as general motivation and expectations about performance in game-like tasks may also mediate the relationship observed here (see Boot, Blakely \& Simons, 2011; Kristjánsson, 2013) although motivational differences have not always been observed (Chisholm \& Kingston, 2015). However it should be noted that in the data presented here, observers were not aware that their performance would be compared with gaming behaviour, since they were only asked about gaming after completing the RSVP task. Hence it cannot be the case that motivation during this particular task was affected by expectations about their gaming characteristics here. Another consideration is that gender differences between VGPs and NVGPs noted by some (Feng, Spence \& Pratt, 2007) may have affected the data here, although we were unable to assess this directly due to the relatively small sample of LVGPs and NVGPs of each gender. Nonetheless with these various factors considered, it remains the case that for the group of very moderate video game players tested here performed better than NVGPs in responding to the critical second targets embedded within an RSVP stream and this is likely due to more efficient perceptual processing of rapidly presented stimuli. 


\section{References}

Adelman, J. S. (2011). Letters in time and retinopic space. Psychological Review, 118(4), 570-582.

Appelbaum, L. G., Cain, M. S., Darling, E. F., \& Mitroff, S. R. (2013). Action video game playing is associated with improved visual sensitivity, but not alterations in visual sensory memory. Attention, Perception, \& Psychophysics,75(6), 1161-1167.

Arnell, K. M., Howe, A. E., Joanisse, M. F., \& Klein, R. M. (2006). Relationships between attentional blink magnitude, RSVP target accuracy, and performance on other cognitive tasks. Memory \& Cognition, 34, 1472-1483. doi:10.3758/BF03195912

Arnell, K. M., \& Jolicoeur, P. (1999). The Attentional Blink Across Stimulus Modalities : Evidence for Central Processing Limitations. Journal of Experimental Psychology. Human Perception and Performance, 25(3), 630-648.

Arnell, K. M., Stokes, K., MacLean, M. H., \& Gicante, C. (2010). Executive control processes of working memory predict attentional blink magnitude over and above storage capacity. Psychological Research, 74, 1-11. doi:10.1007/s00426-008-0200-4

Bavelier, D., Achtman, R. L., Mani, M., \& Föcker, J. (2012). Neural bases of selective attention in action video game players. Vision Research, 61, 132-43. doi:10.1016/j.visres.2011.08.007

Boot, W. R., Kramer, A. F., Simons, D. J., Fabiani, M., \& Gratton, G. (2008). The effects of video game playing on attention, memory, and executive control. Acta Psychologica, 129(3), 387-98. doi:10.1016/j.actpsy.2008.09.005.

Cain, M. S., Landau, A. N., \& Shimamura, A. P. (2012). Action video game experience reduces the cost of switching tasks. Attention, Perception, \& Psychophysics, 74(4), 641-647.

Cain, M. S., Prinzmetal, W., Shimamura, A. P., \& Landau, A. N. (2014). Improved control of exogenous attention in action video game players. Frontiers in Psychology, 5.

Carrasco, M. \& McElree, B. (2001). Covert attention speeds the accrual of visual information. Proceedings of the National Academy of Sciences of the United States of America, 98, 5363-5367.

Castel, A. D., Pratt, J., \& Drummond, E. (2005). The effects of action video game experience on the time course of inhibition of return and the efficiency of visual search. Acta Psychologica, 119(2), 217-30. doi:10.1016/j.actpsy.2005.02.004

Chisholm, J. D., \& Kingstone, A. (2012). Improved top-down control reduces oculomotor capture: The case of action video game players. Attention, Perception, \& Psychophysics, 74(2), 257-262.

Chisholm, J. D., \& Kingstone, A. (2015). Action video game players' visual search advantage extends to biologically relevant stimuli. Acta psychologica,159, 93-99.

Chun, M. M., \& Potter, M. C. (1995). A Two-Stage Model for Multiple Target Detection in Rapid Serial Visual Presentation. Journal of Experimental Psychology: Human Perception and Performance, 21(1), 109-127.

Chun, M. M., \& Potter, M. C. (2001). The attentional blink and task switching within and across modalities. In K. Shapiro (Ed.), The limits of attention: Temporal constraints on human information processing (Vol. 37240, pp. 20-35). New York, NY, US: Oxford University Press. 
Colzato, L. S., Spapé, M. M. A., Pannebakker, M. M., \& Hommel, B. (2007). Working memory and the attentional blink: Blink size is predicted by individual differences in operation span. Psychonomic Bulletin \& Review, 14, 1051-1057. doi:10.3758/BF03193090

Colzato, L. S., Van Leeuwen, P. J., Van Den Wildenberg, W., \& Hommel, B. (2010). DOOM'd to switch: superior cognitive flexibility in players of first person shooter games. Frontiers in Psychology, 1,8 .

Connolly, T. M., Boyle, E. A., MacArthur, E., Hainey, T., \& Boyle, J. M. (2012). A systematic literature review of empirical evidence on computer games and serious games. Computers \& Education, 59(2), 661-686. doi:10.1016/j.compedu.2012.03.004

Crawford, G., \& Gosling, V. K. (2009). More Than a Game : Sports-Themed Video Games and Player Narratives. Sociology of Sport Journal, 26, 50-66.Donohue, S. E., Woldorff, M. G., \& Mitroff, S. R. (2010). Video game players show more precise multisensory temporal processing abilities. Attention, Perception, \& Psychophysics, 72(4), 1120-1129.

Duncan, J., Martens, S., \& Ward, R. (1997). Restrictedattentional capacity within but not between sensory modalities. Nature, 387(6635), 808-10.

Dux, P. E., \& Marois, R. (2008). Distractor inhibition predicts individual differences in the attentional blink. PLoS One, 3, e3330. doi:10.1371/journal.pone.0003330

Dux, P., \& Marois, R. (2010). How humans search for targets through time: A review of data and theory from the attentional blink. Attention, Perception \& Psychophysics, 71(8), 1683-1700. doi:10.3758/APP.71.8.1683.How

Dye, M. W. G., \& Bavelier, D. (2010). Differential development of visual attention skills in schoolage children. Vision Research, 50(4), 452-459. doi:10.1016/j.visres.2009.10.010.

Dye, M. W. G., Green, C. S., \& Bavelier, D. (2009a). Increasing Speed of Processing With Action Video Games. Current Directions in Psychological Science, 18(6), 321-326. doi:10.1111/j.14678721.2009.01660.

Dye, M. W. G., Green, C. S., \& Bavelier, D. (2009b). The development of attention skills in action video game players. Neuropsychologia, 47(8-9), 1780-1789.

doi:10.1016/j.neuropsychologia.2009.02.002.

Enns, J. T., Austen, E. L., Di Lollo, V., Rauschenberger, R., \& Yantis, S. (2001). New objects dominate luminance transients in setting attentional priority. Journal of Experimental Psychology: Human Perception \& Performance, 27, 1287-1302.

Feng, J., Spence, I., \& Pratt, J. (2007). Playing an action video game reduces gender differences in spatial cognition. Psychological Science, 18(10), 850-5. doi:10.1111/j.1467-9280.2007.01990.

Gentile, D. a., Swing, E. L., Lim, C. G., \& Khoo, A. (2012). Video game playing, attention problems, and impulsiveness: Evidence of bidirectional causality. Psychology of Popular Media Culture, 1(1), 62-70. doi:10.1037/a0026969

Giesbrecht, B., \& Di Lollo, V. (1998). Beyond the attentional blink: visual masking by object substitution. Journal of Experimental Psychology: Human Perception \& Performance, 24, 1454-1466. 
Green, C. S., \& Bavelier, D. (2003). Action video game modifies visual selective attention. Nature, 423(May), 534-537.

Green, C. S., \& Bavelier, D. (2006a). Effect of action video games on the spatial distribution of visuospatial attention. Journal of Experimental Psychology. Human Perception and Performance, 32(6), 1465-78. doi:10.1037/0096-1523.32.6.1465

Green, C. S., \& Bavelier, D. (2006b). Enumeration versus multiple object tracking: the case of action video game players. Cognition, 101(1), 217-45. doi:10.1016/j.cognition.2005.10.004

Green, C. S., \& Bavelier, D. (2007). Action-Video-Game Experience Alters the Spatial Resolution of Vision. Psychological Science, 18(1), 88-95.

Green, C. S., \& Bavelier, D. (2012). Learning, attentional control, and action video games. Current Biology: $C B, 22(6)$, R197-206. doi:10.1016/j.cub.2012.02.012

Green, S. C., Sugarman, M. A., Medford, K., Klobusicky, E., \& Bavelier, D. (2012). The effect of action video game experience on task-switching. Computers in Human Behavior, 28(3), 984-994.

Guest, D., Howard, C. J., Brown, L. \& Gleeson, H. (2015). Aging and the rate of visual information processing. Journal of Vision.

Guest, D, \& Lamberts, K. (2010). The prioritisation of perceptual processing in categorisation. Attention, Perception \& Psychophysics, 72, 1079-1096.

Guest, D, \& Lamberts, K. (2011). The time course of similarity effects in visual search. Journal of Experimental Psychology; Human Perception and Performance, 37, 1667-1688.

Hubert-Wallander, B., Green, C. S., Sugarman, M., \& Bavelier, D. (2011). Changes in search rate but not in the dynamics of exogenous attention in action videogame players. Attention, Perception \& Psychophysics, 73(8), 2399-412. doi:10.3758/s13414-011-0194-7.

Howard, C. J. \& Holcombe, A. O. (2008). Tracking the changing features of multiple objects: progressively poorer perceptual precision and progressively greater perceptual lag. Vision Research, $48,1164-1180$.

Jannati, A., \& Spalek, T. M. \& Di Lollo, V. (2011). Neither backward masking of T2 nor task switching is necessary for the attentional blink. Psychonomic Bulletin \& Review, 18:70-75.

Kelly, A. J., \& Dux, P. E. (2011) Different attentional blink tasks reflect distinct information processing limitations: an individual differences approach. Journal of Experimental Psychology: Human Perception and Performance, 37, 6, 1867.

Kristjánsson, Á. (2013). The case for causal influences of action videogame play upon vision and attention. Attention, Perception, \& Psychophysics, 75(4), 667-672.

Li, R., Polat, U., Scalzo, F., \& Bavelier, D. (2010). Reducing backward masking through action game training. Journal of Vision, 10(14):33, 1-13.

Luck, S. J., Hillyard, S. A., Mouloua, M., Woldorff, M. G., Clark, V. P. \& Hawkins, H. L. (1994). Effects of spatial cuing on luminance detectability: psychophysical and electrophysiological evidence for early selection. Journal of Experimental Psychology: Human Perception \& Performance, 20(4), 887. 
Luck, S. J., Vogel, E.K., Shapiro, K. L. (1996). Word meanings can be accessed but not reported during the attentional blink. Nature, 383 (6601):616-618.

Maclin, E. L., Mathewson, K. E., Low, K. a, Boot, W. R., Kramer, A. F., Fabiani, M., \& Gratton, G. (2011). Learning to multitask: effects of video game practice on electrophysiological indices of attention and resource allocation. Psychophysiology, 48(9), 1173-83. doi:10.1111/j.14698986.2011.01189.

Martens, S., \& Valchev, N. (2009). Individual differences in the attentional blink: The important role of irrelevant information. Experimental Psychology, 56, 18-26. doi:10.1027/1618-3169.56.1.1

Martens, S., \& Wyble, B. (2010). The attentional blink: past, present, and future of a blind spot in perceptual awareness. Neuroscience and Biobehavioral Reviews, 34(6), 947-57. doi:10.1016/j.neubiorev.2009.12.005

Mishra, J., Zinni, M., Bavelier, D., \& Hillyard, S. A. (2011). Neural basis of superior performance of action videogame players in an attention-demanding task. The Journal of Neuroscience, 31(3), 992-8. doi:10.1523/JNEUROSCI.4834-10.2011

Murphy, K., \& Spencer, A. (2009). Playing video games does not make for better visual attention skills. Journal of Articles in Support of the Null Hypothesis, 6(1), 1-20.

Nieuwenstein, M. R., Potter, M. C., \& Theeuwes, J. (2009). Unmasking the attentional blink. Journal of Experimental Psychology. Human Perception and Performance, 35(1), 159-69. doi:10.1037/00961523.35.1.159.

Oei, A. C. \& Patterson, M. D. (2013). Enhancing Cognition with Video Games: A Multiple Game Training Study. PLoS ONE 8(3): e58546. doi:10.1371/

Oei, A. C. \& Patterson, M. D. (2015). Enhancing perceptual and attentional skills requires common demands between the action video games and transfer tasks. Frontiers in Psychology, 6, 113, doi: 10.3389/fpsyg.2015.00113

Olivers, C. N. L., \& Meeter, M. (2008). A boost and bounce theory of temporal attention. Psychological Review, 115(4), 836-863.Orvis, K. a., Horn, D. B., \& Belanich, J. (2009). An examination of the role individual differences play in videogame-based training. Military Psychology, 21(4), 461-481. doi:10.1080/08995600903206412

Pasch, M., Bianchi-Berthouze, N., van Dijk, B., \& Nijholt, A. (2009). Movement-based sports video games: Investigating motivation and gaming experience. Entertainment Computing, 1(2), 49-61. doi:10.1016/j.entcom.2009.09.004

Pohl, C., Kunde, W., Ganz, T., Conzelmann, A., Pauli, P., \& Kiesel, A. (2014). Gaming to see: action video gaming is associated with enhanced processing of masked stimuli. Frontiers in psychology, 5.

Raymond, J. E., Shapiro, K. L., \& Arnell, K. M. (1992). Temporary suppression of visual processing in an RSVP task: An attentional blink? Journal of Experimental Psychology: Human Perception and Performance, 18(3), 849-860.

Strobach, T., Frensch, P. A, \& Schubert, T. (2012). Video game practice optimizes executive control skills in dual-task and task switching situations. Acta Psychologica, 140(1), 13-24.

doi:10.1016/j.actpsy.2012.02.001. 
Sungur, H., \& Boduroglu, A. (2012). Action video game players form more detailed representation of objects. Acta Psychologica, 139(2), 327-334.

Taatgen, N. A., Juvina, I., Schipper, M., Borst, J. P., \& Martens, S. (2009). Too much control can hurt: A threaded cognition model of the attentional blink. Cognitive Psychology, 59(1), 129.Unsworth, N., Redick, T. S., McMillan, B. D., Hambrick, D. Z., Kane, M. J. \& Engle, R. W. (2015). Is Playing Video Games Related to Cognitive Abilities? Psychological Science, 26(6) 759-774.

Vallett, D. B., Lamb, R. L., \& Annetta, L. (2013). The gorilla in the room: The impacts of video-game play on visual attention. Computers in Human Behavior, 29(6), 2183-2187. doi:10.1016/j.chb.2013.05.001

Vogel, E. K., Luck, S. J., Shapiro, K. L. (1998). Electrophysiological evidence for a post-perceptual locus of suppression during the attentional blink. Journal of Experimental Psychology: Human Perception and Performance, 24:1656-1674.

Westwood, D., \& Griffiths, M. D. (2010). The Role of Structural Characteristics in Video-Game. Cyberpsychology, Behavior and Social Networking, 13(5), 581-585.

Wyble, B., Bowman, H., \& Nieuwenstein, M. (2009). The attentional blink provides episodic distinctiveness: Sparing at a cost. Journal of Experimental Psychology: Human Perception and Performance, 35(3), 787-807. 